\title{
Estudio sobre las redes sociales personales y las redes sociales virtuales en la cibercultura adolescente actual
}

\section{Study of personal social networks and virtual social networks in the current teenage cyberculture}

\author{
Correa María Soledad, Vitaliti José Maria \\ Facultad de Psicología, Universidad del Aconcagua (UDA), INCIHUSA, Consejo Nacional de Investigaciones \\ científicas y Tecnológicas (CONICET). \\ (Rec: marzo de 2018 - Acept: octubre de 2018)
}

\begin{abstract}
Resumen
En la actualidad, las plataformas virtuales (Facebook, Instagram, Twitter, entre otras) han impactado en la cotidianeidad generando cambios en las interacciones personales y los patrones de relación. Generalmente, los usuarios de estas plataformas son adolescentes donde lo virtual se hace parte de la construcción de identidad y pertenencia al grupo de pares. El objetivo del trabajo es comprender las dinámicas de la cibercultura adolescente a través de la indagación en redes personales y virtuales de cuatro adolescentes de Mendoza-Argentina.
\end{abstract}

Se escogió el estudio de caso, seleccionándose 4 adolescentes de entre 15 y 17 años. Las herramientas utilizadas fueron: cuestionario administrado a través del software Egonet, observación de la plataforma virtual que utiliza el participante y entrevista semidirigida como modalidad corroborativa de los datos.

Los resultados aportan reflexiones para reflexionar en la cibercultura adolescente, resultando arduo diferenciar lo virtual de lo cara a cara y/o online de lo offline. Existe una gran interacción de los adolescentes en las redes virtuales, pueden identificar a sus seguidores y escoger las maneras de exponerse socialmente. Los familiares tienen un rol secundario, tanto en las redes personales y virtuales. Por último, las plataformas virtuales Instagram y Snapchat tienen mayor protagonismo entre las indagadas.

Palabras clave: Adolescencia, redes sociales virtuales, redes sociales personales, análisis de redes sociales

\begin{abstract}
Nowadays, virtual platforms (such as Facebook, Instagram, and Twitter, amongst others) have a great impact on everyday life, generating changes in people's interactions and relationship patterns. Usually, teenagers are the main users of these platforms, where the virtual becomes part of the construction of identity and peer group dynamics. The objective of this study was to understand the dynamics of teenage cyberculture through the investigation of personal and virtual networks of four teenagers in Mendoza, Argentina. A case study method was chosen, where four adolescents between 15 and 17 years old were chosen as participants. The study applies the following tools: a questionnaire administered through the Egonet software, the observation of the social network most frequently used by the subject and a semi-structured interview. The results lead us to reflect on a teen cyberculture in which peer relationships are virtualized, making it difficult to differentiate between online and offline. There is a great interaction between teenagers in virtual networks, where they can identify their followers and choose the way in which they socially expose themselves. Family bonds play a secondary role in both personal and virtual social networks. Furthermore, Instagram and Snapchat were found to be more popular amongst teenagers, rather than the others.
\end{abstract}

Keywords: Adolescence, virtual social networks, personal social networks, social network analysis 


\section{Introducción}

En la actualidad, las redes sociales virtuales (Facebook, Instagram, Twitter, entre otras) han impactado en la cotidianeidad de las personas, generando "un estilo de relación casi independiente de los lugares geográficos y de la coincidencia de los tiempos (...) aunque no se le pueda fijar en ninguna coordenada espacio-temporal, lo virtual es sin embargo real" (Levy, 2007).

Este fenómeno se vio impulsado por los dispositivos móviles o smartphones, a través de los cuales los usos son diversos y múltiples. Ortega Gutiérrez y Ricaurte Quijano (2010) nombran algunos de ellos como: la expresión de emociones y afectos, la performance de las identidades múltiples y versátiles, el establecimiento o re-establecimiento de contactos, la construcción de una imagen pública, el entretenimiento y la organización civil, entre otras. De esta manera, vemos que la diversidad de atractivos de estas redes contribuye a su popularidad.

\section{Ciberculturas adolescentes}

Este desarrollo material, donde hay tecnología que nos permite estar conectados por Internet con diversas personas del mundo (Evans, 2011), forma parte de una retroalimentación social y cultural que se llama cibercultura. La cibercultura es definida por Levy (2007) como "el conjunto de las técnicas (materiales e intelectuales), de prácticas, de actitudes, de modos de pensamiento y de los valores que se desarrollan conjuntamente en el crecimiento del ciberespacio" (Levy, 2007). Puntualizando en la importancia de la cibercultura para la etapa de la juventud, Urresti (2008) describe el concepto de "ciberculturas juveniles" como "ámbitos de encuentro virtual entre jóvenes que, a pesar de la distancia geográfica, intercambian información sobre cuestiones de su interés, se relacionan entre sí y se comunican a diario" (2008: 10). A partir de las ciberculturas juveniles "se forjan una imagen de sí mismos, de los grupos a los que pertenecen, y de esa manera toman conciencia de la generación en la que se incluyen y el mundo que los rodea" (Urresti, 2008: 10). Cibercultura definida por Levy (2007) hace hincapié en los sistemas culturales que acompañan a las tecnologías digitales, mientras que la cibercultura juvenil de Urresti (2008) apunta a que los jóvenes forjen su identidad a partir de la interrelación virtual cotidiana. Estos autores no establecen diferenciaciones entre juventud y adolescencia al hablar de "cultura juvenil"; sin embargo, parece relevante profundizar en las características particulares de la adolescencia como etapa vital, y que deben considerarse a la hora de analizar las relaciones de este grupo etáreo.

La adolescencia comprende una etapa del desarrollo humano que implica cambios biológicos, psicológicos y sociales. A su vez, cada sociedad, época y sector social, construye las formas culturales e institucionales que influyen en la adolescencia, hasta el inicio de la etapa "adultez-joven" (Margulis, 2004: 6). La adolescencia se diferencia de la juventud por la dependencia socio-emocional parental, la construcción de la identidad y la pertenencia al grupo de pares.

Sociológicamente hablando, Giró (2011) refiere que la construcción de la identidad adolescente se lleva a cabo a través de procesos de mediación e intervención del otro que en sí mismos son significativos gracias a la relación dialógica que se establece entre adolescentes. Perteneciendo y adscribiéndose al gru- po de pares, eliminan toda posibilidad de rechazo o exclusión, lo que implica una ventaja social inestimable y superior a la seguridad que se puede hallar en el mundo de adulto (2011: 79).

La adolescencia nos brinda enfoques diferenciados al vincularlas con la virtualidad, pudiendo de esta manera, hablar de una cibercultura adolescente. Algunos estudios abonan esta conceptualización, como los de: Paredes, Vitaliti, Strafile, Aguirre, y Jara, (2013) quienes realizan un estudio sobre redes sociales virtuales (Facebook) de adolescentes de contexto rural en Mendoza-Argentina, donde la totalidad de estos usa esta red virtual. Además, los estudiantes refirieron que Facebook sirve para reforzar el apoyo informacional (intercambio de mensajes), tangible (intercambio de elementos concretos) $y$ afectivo (intercambio emocional), pero no tanto el apoyo axiológico (intercambio de valores) (2013: 115). En un estudio similar realizado, el mismo grupo de estudios de Paredes, Aguirre, Vitaliti, Strafile, Jara y Sottile (2015) concluyen que las redes personales y sus distintos tipos de apoyo se han digitalizado, fortaleciendo vínculos por medios tecnológicos sin que esto implique contacto físico (Paredes et al., 2015: 157).

Ak, Koruklu y Yılmaz (2012) investigaron sobre el uso de internet por parte de los estudiantes en relación con el género y con el puntaje del Test de Adicción a Internet. Se observa que, sin importar el género, se utiliza internet predominantemente para el uso de Facebook para comunicarse online entre pares.

Courtois, All y Vanwynsberghe (2012) indican que las redes sociales son fuentes valiosas de información para relaciones offline. Los resultados confirman cómo Internet refuerza la habilidad de comunicarse mediante el sostén de lazos fuertes (amigos cercanos) y lazos débiles (conocidos), permitiendo el intercambio de información y proveyendo apoyo social en las relaciones (2012: 293). En la misma línea, Linne expone que para los adolescentes la red social es su entorno central de entretenimiento y comunicabilidad (2014). La información sobre el uso de redes sociales virtuales en adolescentes nos dice que principalmente se utilizan para la autopresentación, el intercambio de contenidos personales entre amistades, la búsqueda de relaciones sexo-afectivas y la exploración de distintos aspectos de su sociabilidad e identidad (Linne, 2014)

En cuanto a rasgos de personalidad y comportamiento online, Peter, Valkenburg y Schouten (2005) expresaron que el grado de introversión o extraversión de los adolescentes no influencia directamente la formación de amistades online. No obstante, la introversión o extroversión se diferencia en la auto-exposición online, la frecuencia de comunicación, y el motivo de compensación social, mediadores que sí influyen en la conformación de este tipo de amistades. (2005: 428-429).

A partir de estos estudios podemos destacar que: i. los/as adolescentes utilizan internet predominantemente en sus redes sociales (Facebook); ii. tienen mayor posibilidad de sentirse apoyados desde lo informacional, tangible y emocional; iii. las relaciones se encuentran mediadas por la virtualidad y por último, iv. la intro/extraversión del usuario se diferencia por la autoexposición y la compensación social.

\section{Redes personales (RP) versus redes virtuales (RV)}

Las redes personales son el conjunto de relaciones personales a través de las cuales las personas formamos parte de estructuras sociales mayores (Casquero, 2013). La composición 
y la estructura de la red personal reflejan un momento dado en la vida de una persona con determinados roles en la estructura socio-económica, en el marco de un conjunto de instituciones culturales y en un momento histórico determinado (Molina, Lerner, y Gómez, 2008).

Desde nuestro punto de vista, la red personal es una condensación relacional dinámica de personas. Dicha red está determinada por distintos factores tales como subjetividad, cotidianeidad e intercambio entre los miembros. A su vez, están dinamizadas por la estructura cultural, social, histórica y económica que las contiene.

Por otro lado, se entiende red virtual como una interconexión entre usuarios a través de una plataforma virtual (Facebook, Twitter, Instagram, entre otras). Castañeda y Gutiérrez (2010) expresan que las redes sociales -virtuales- son "aquellas herramientas telemáticas de comunicación que tienen como base a la Web 2.0" (2010: 1). Estas herramientas se organizan alrededor de perfiles personales o profesionales de los usuarios y tienen como objetivo conectar secuencialmente a los propietarios de dichos perfiles a través de categorías, grupos, etiquetados personales, etc., ligados a su propia persona o perfil profesional (Castañeda y Gutiérrez, 2010). Las mismas se asemejan a una red analógica en el sentido de que son puntos conectados en el espacio, pero de manera virtual, intangible.

Las plataformas virtuales presentan una diferenciación en cuanto a la comunidad a la que se dirigen y la mediatización que proporcionan a través de lo virtual. Sin embargo, Courtois afirma que "dicotomizar rígidamente entre online y offline se ha vuelto cada vez más difícil. Lo que sucede online tiene implicancias offline y viceversa" (Courtois, Anissa y Vanwynsberghe, 2012). Vidales-Bolaños y Sádaba- Velazquer han estudiado esta interacción entre lo virtual y lo real, concluyendo que "la comunicación mediada por el móvil influye de forma positiva en las relaciones sociales de aquellos adolescentes que se muestran más capaces de conciliar sus actividades dentro y fuera de la Red" (2017: 25-26).

A continuación, se propone una breve reseña de plataformas virtuales populares actualmente, con el fin de establecer las diferencias de uso de cada una de ellas:
Facebook: este sitio fue fundado en febrero de 2004 por Mark Elliot Zuckerberg. Tiene el objetivo de comunicar y compartir entre los usuarios de la plataforma, usando distintos recursos.

Instagram: esta plataforma tecnológica se conceptualiza a sí mismo como "una forma sencilla de capturar y compartir los mejores momentos con amigos, familiares y con el mundo" y continúa diciendo que "...actualmente cuenta con más de 400 millones de usuarios" (Instagram Inc., 2016). Entre las características particulares de esta plataforma, se nomina la capacidad de editar los fotos y videos con distintas herramientas y filtros; la facilidad para encontrar personas a las que seguir en función de gustos personales y mantenerse al día con las fotos y videos de usuarios alrededor del mundo; la posibilidad de compartir las publicaciones a otras redes sociales virtuales como Facebook, Twitter y Tumblr, y por último, la posibilidad de enviar mensajes con fotos y videos por mensaje privado. Particularmente en el caso de adolescentes, Jang, Han, Shih y Lee (2015) encontraron que tienden a publicar menor cantidad de fotos, pero sí a utilizar gran cantidad de etiquetas e interactuar bastante a través de likes y comentarios. Además, suelen publicar mayor cantidad de selfies y expresarse más, demostrando un mayor sentido de auto-presentación (Jang et al., 2015).

Snapchat: es una aplicación que permite a los usuarios tomar fotografías, grabar vídeos, añadir textos y dibujos y enviarlos a una lista de contactos limitados o publicarlos en su "historia" , la cual desaparece después de veinticuatro horas. Estos vídeos y fotografías se conocen como "Snaps" y los usuarios pueden controlar el tiempo durante el que estos serán visibles (de 1 a 10 segundos de duración), tras lo cual desaparecerán de la pantalla del destinatario y serán borrados del servidor de Snapchat (Snap Inc., 2016).

Twitter: la plataforma se describe a sí misma como "la mejor forma de conectar, de poder expresarte y descubrir que está pasando (...) permite conectarse con personas y con intereses propios para tener un acceso sin filtrar y perspectivas únicas tras bambalinas $y$, además, permite expresarse con fotos, videos y comentarios" (Twitter Inc., 2016).

A continuación (Tabla $n^{\circ} 1$ ), se realiza una síntesis con las principales características de las plataformas virtuales teniendo presente las características diferenciadoras y comunes entre ellas.

Tabla No1

Elementos vinculantes entre Facebook, Instagram, Snapchat y Twitter

\begin{tabular}{|c|c|c|c|c|}
\hline Características & Facebook & Instagram & Snapchat & Twitter \\
\hline Objetivo & $\begin{array}{l}\text {-Compartir contenidos } \\
\text { - Conectar con los } \\
\text { "amigos" previamente } \\
\text { aceptados }\end{array}$ & $\begin{array}{c}\text { - Compartir momentos a } \\
\text { través de fotos }\end{array}$ & $\begin{array}{l}\text { - Intercambiar Snaps } \\
\text { entre contactos o publi- } \\
\text { carlos en la historia. }\end{array}$ & $\begin{array}{c}\text { - Conectar, posibilidad de } \\
\text { comentar y compartir }\end{array}$ \\
\hline Nominación del otro & Amigos & Seguidores/Seguidos & Seguidores/ seguidos & Siguiendo/ seguidores \\
\hline Chat o mensajería directa & Sí & Sí & Sí & Sí \\
\hline Me gusta & Sí. & Sí & No & Sí \\
\hline Comentarios & Sí & Sí & $\begin{array}{l}\text { No, solo a través de con- } \\
\text { versaciones privadas }\end{array}$ & $\begin{array}{l}\text { Sí, en forma de respues- } \\
\text { tas }\end{array}$ \\
\hline Etiquetas en fotos & Sí & Sí & No & Sí \\
\hline Etiquetas a otros usuarios & $\begin{array}{l}\text { Sí, en comentarios y } \\
\text { publicaciones }\end{array}$ & Sí, en comentarios & No & Sí \\
\hline
\end{tabular}

Fuentes: Instagram Inc (2017); Twitter Inc. (2016); Snap Inc. (2016); Facebook @ (2017). 
En cuanto las investigaciones actuales sobre psicología y plataformas virtuales, Vanessa Pérez afirma que se hace necesario "diversificar los estudios para abarcar las nuevas formas de interacción como Snapchat o Instagram, que suelen atraer a un perfil diferente de Facebook (por lo general, adolescentes)" (2018: 100), junto a ello expresa que "se ha avanzado desde las investi ggaciones iniciales acerca de la construcción de la identidad online de los usuarios de la red a los estudios sobre la utiliza $\neg$ ción masiva de los selfies debido al creciente uso de redes sociales con un alto componente de exposición personal como Instagram" (Pérez, 2018: 103). De esta manera se observa la necesidad de investigar plataformas virtuales diferenciadas de Facebook y twitter, ya que hay mayor uso por parte de adolescentes.

En base a lo anteriormente expuesto, el presente trabajo se propone comprender las dinámicas de la cibercultura adolescente a través de la indagación en las redes personales y virtuales de cuatro adolescentes de Mendoza-Argentina. En este sentido pretendemos: identificar las plataformas virtuales mayormente utilizadas por los participantes del estudio y por los miembros de sus redes personales con el fin de presentar las formas de comunicación utilizadas en la actualidad; describir las valoraciones que realizan los adolescentes sobre sus redes virtuales y redes personales para establecer los modos de pensar estos medios; especificar las características composicionales y estructurales en la red personal de los participantes para indagar las tendencias sociales relacionadas con la cibercultura adolescentes; y por último, relacionar las características que tienen las centralidades en la red virtual y red personal del/a adolescentes participantes, con el fin de realizar comparaciones entre los sujetos conocidos personalmente y conocidos únicamente a través de la plataforma virtual.

\section{Metodología}

La metodología utilizada es mixta o cuali-cuantitativa. El tipo de estudio es estudio de caso, el cual se orienta a recabar datos por participante desde una variedad de fuentes datos guiados por las preguntas de investigación (Martínez Carazo, 2006) cuyo alcance del estudio es exploratorio-descriptivo. El diseño es fenomenológico y tiene el propósito de explorar, describir y comprender las experiencias de las personas con respecto a un acontecimiento y descubrir los elementos en común de tales vivencias (Hernández Sampieri, Fernandez-Collado y Baptista Lucio, 2014).

\section{Participantes}

Los participantes de la investigación son cuatro adolescentes de la provincia de Mendoza Argentina. Se comenzó por dos al principio y a los siguientes por método de bola de nieve (Hernández Sampieri et al. 2014). Los adolescentes tenían entre 15 a 17 años de edad, dos de sexo femenino y dos de sexo masculino. Las pertenencias locales de los participantes y estratos sociales eran diferentes aunque no se utilizó como dato relevante para el estudio.

Tabla $\mathrm{N}^{\circ} 2$

Edad y género de los participantes.

\begin{tabular}{ccc}
\hline Participante & Edad & Género \\
\hline 1 & 16 & Femenino \\
2 & 15 & Masculino \\
3 & 16 & Masculino \\
4 & 17 & Femenino \\
\hline
\end{tabular}

\section{Instrumentos}

En el estudio se utilizaron tres instrumentos de recolección de datos con distintas modalidades de análisis.

Tabla $\mathrm{N}^{\circ} 3$

Conceptos indagados, instrumento de recolección y análisis de los datos.

\begin{tabular}{ccc}
\hline $\begin{array}{c}\text { Conceptos } \\
\text { indagados }\end{array}$ & $\begin{array}{c}\text { Instrumento de } \\
\text { recolección }\end{array}$ & Análisis \\
\hline Red personal & $\begin{array}{c}\text { Cuestionario a } \\
\text { partir del software } \\
\text { Egonet }\end{array}$ & $\begin{array}{c}\text { Estadísticos } \\
\text { reticulares a } \\
\text { partir del software } \\
\text { Egonet. } \\
\text { Red virtual }\end{array}$ \\
& Observación & $\begin{array}{c}\text { Construcción de } \\
\text { matriz en excel y } \\
\text { gráfico de red a } \\
\text { través del }\end{array}$ \\
Usuario & Entrevista & $\begin{array}{c}\text { Software Ucinet. } \\
\text { contisis de }\end{array}$ \\
(valoraciones) & semidirigida & contenido \\
\hline
\end{tabular}

Cuestionario: se trata de un cuestionario realizado por el investigador/a basándose en el software Egonet, programa especializado en análisis de redes personales egocentradas (Ávila Molera, 2015). Este cuestionario consta de cuatro partes:

Primero: aborda preguntas sobre el participante, las cuales en este caso fueron: edad, género y plataformas virtuales utilizadas. En esta última categoría se incluyó a Facebook, Instagram, Twitter y Snapchat.

Segunda: se solicita al sujeto que nombre 35 personas (alters) con las cuales haya dialogado en la última semana. Se ha limitado a 35 personas ya que es un mínimo de alters que pueden aportan una idea suficientemente aproximada de la estructura de la red social personal (McCarty, 2010)

Tercera: las preguntas van dirigidas al participante sobre las personas que nombró. Se consultó sobre: edad, género, redes sociales virtuales utilizadas y rol ocupado por el alter. En cuanto al rol del alter se podía elegir entre las siguientes opciones: familiar conviviente, familiar no conviviente, amigo, compañero de escuela, compañero de una actividad de tiempo libre, pareja, vecino y otros.

Cuarta: Se consulta al/la entrevistado/a la relación existente entre los alters, a través de la siguiente pregunta: "Alter1 y Alter2, ¿se relacionarían si Ud. no estuviese presente?"

A partir de la toma del instrumento, el Egonet ofrece un gráfico y estadísticos sobre la red personal. Los estadísticos muestran porcentajes de la edad, género, red virtual y rol ocupado de la red personal en general.

Observación no participante de la plataforma virtual: se solicitó al participante la posibilidad de observar la plataforma virtual utilizada con mayor frecuencia. A partir de ello, se observó por un tiempo acotado las publicaciones (fotos o comentarios) y la interacción a través de me gusta y comentarios en las mismas. El tiempo de observación de la plataforma virtual fue retrospectivo y el objetivo fue recorrer entre 4-6 publicaciones. A continuación, se presenta un cuadro que describe 
-por participante- la plataforma virtual utilizada, la cantidad de publicaciones, la distancia temporal entre publicaciones, la

cantidad de me gusta (MG) y comentarios en total.

Tabla $\mathrm{N}^{\circ} 4$

Datos relevantes sobre el seguimiento de la Red virtual

\begin{tabular}{|c|c|c|c|c|c|}
\hline Participante & $\begin{array}{c}\text { Red virtual } \\
\text { analizada }\end{array}$ & Distancia temporal & Publicaciones & $\begin{array}{c}\text { Cantidad de me } \\
\text { gustas y } \\
\text { comentarios }\end{array}$ & Seguidores \\
\hline 1 & Instagram & 2 meses & 6 & 648 & 311 \\
\hline 2 & Instagram & 7 meses & 2 & 15 & 11 \\
\hline 3 & Instagram & 3 meses & 5 & 1055 & 485 \\
\hline 4 & Instagram & 4 meses & 5 & 159 & 91 \\
\hline
\end{tabular}

La plataforma virtual que refirieron los participantes fue Instagram, ya que la mayoría de los participantes eran usuarios de esta red. Se escogió trabajar en las publicaciones, entendiéndolas como "un hecho virtual", en el que el usuario toma protagonismo en la plataforma e interactúa con los seguidores/amigos.

A partir de la observación de la actividad en la plataforma virtual se construyó la red virtual. En primer lugar, se realizó una matriz de modo dos en las que se ubicó en las columnas a los MG y comentarios por publicación y en las filas los seguidores/amigos. Por cada MG o comentario dado a una publicación del participante de la investigación se colocaba un 1 y el nombre del seguidor/amigo. Luego, mediante el software Ucinet que cuenta con una herramienta llamada NetDraw, se construyó el gráfico de la red virtual.

Entrevista semidirigida: el objetivo fue complementar la información recabada a través de ambas redes (virtual-personal) con las valoraciones que los adolescentes realizan sobre la construcción gráfica o grafo de sus redes (personal o virtual).

\section{Procedimiento}

En un primer lugar se identificaron los primeros participantes para la tarea investigativa, a quienes se les explicaron los objetivos de la investigación, la cantidad de encuentros personales (dos encuentros) y se procedió a la firma de un consentimiento informado por parte de los padres y asentimiento por parte de los adolescentes.

En el primer encuentro se administró el cuestionario construido con el software Egonet. Luego se solicitó la autorización para observar la interacción en la plataforma virtual donde es usuario el participante.

En segundo lugar, los investigadores accedieron a la plataforma virtual del participante, a través de la solicitud de seguimiento (Instagram) y/o la solicitud de amistad (Facebook), según correspondiera. Se decidió construir un perfil que fuera utilizado para los fines de la investigación y terminando el trabajo, se borraría el usuario. Posteriormente, se utilizaron estos datos para la construcción de matriz y de esta manera poder graficar la red utilizando Ucinet.

En tercer lugar, se realizó el análisis de la red virtual y red personal de los participantes, con cuya información se construyeron preguntas particulares y generales a concretarse en la entrevista semidirigida.
Finalmente, se entrevistó a los/as participantes en un segundo encuentro, para trabajar en sus valoraciones de las RV-RP en relación con los nodos centrales, profundizando también en el uso de plataformas virtuales en general y en datos particulares observados a partir de análisis previo de las redes realizado por los investigadores.

\section{Resultados}

Los resultados indagaron la cibercultura adolescente, a partir de cuatro casos en los que se estudiarán las interacciones reticulares. A su vez, se presentarán las valoraciones y usos de los participantes en relación a las plataformas virtuales, la composición y estructura de las redes personales, la descripción de las redes virtuales a partir de la observación de la actividad en las plataformas virtuales más utilizadas y por último, los nodos con mayor nivel interacción tanto de la red personal como de la red virtual.

\section{Valoraciones de los participantes sobre el uso de las pla- taformas virtuales.}

En primer lugar, los/as participantes de la investigación, manifestaron la importancia de estar conectados a través de las redes sociales virtuales. Esta conexión se refiere a los pares e inclusive con quienes se encuentran distanciados geográficamente. Esto se puede observar en las siguientes frases: "Es lo que usamos, o sea, lo que yo uso para todo. Si quiero hablarle a alguien, si quiero ver algo, es lo que uso" [P.1]; "Ponele que queremos hacer algo y más que nada, nos organizamos por redes" [P.3]; "Porque yo creo que, sin ellas, no sé si decirte que estaría totalmente aislada, pero, o sea, como que te une bastante, o sea, con mis primos, por ejemplo, yo no los veo. Si no fuera por las aplicaciones como Instagram y Snapchat yo ni hablaría con ellos" [P.4]; "Estar conectado, siempre actualizado" [P.3].

Asimismo, según los participantes, la rapidez y sencillez son características relevantes del estar conectado, las que pensarse como eficiencia y eficacia: "es mucho más fácil. O sea, es mucho más rápido todo" [P.1]. Rapidez podría entenderse como celeridad del circuito mismo de las plataformas virtuales, por ejemplo, se envía un mensaje, se corrobora que llegó a destino y además que ha sido leído por el receptor. Aunque también como característica contemporánea, donde la temporalidad se ha desplazado hacia la inmediatez. Por último, fue relevante para los participantes la variedad de información transmitida mediante las redes virtuales. En la entrevista lo manifiestan así: "Compartimos cosas, fotos, comentarios, archivos" [P.1]. 
El uso de las plataformas virtuales en las redes personales. En primer lugar, cuando hablamos de uso nos referimos a la mención de los participantes sobre la filiación a alguna plataforma virtual. A continuación se presentan las plataformas usadas por los participantes.

Tabla $\mathrm{N}^{\circ} 5$

Participantes y plataformas virtuales utilizadas.

\begin{tabular}{cc}
\hline Participantes & Plataformas virtuales \\
\hline 1 & $\begin{array}{c}\text { Instagram, Facebook, } \\
\text { Snapchat, Twitter } \\
2\end{array}$ \\
3 & Instagram, Facebook \\
4 & Instagram, Snapchat \\
\hline
\end{tabular}

Referencias: Instagram; Facebook; Snapchat; Twitter.

En la tabla $N^{\circ} 5$ la plataforma virtual mayormente utilizada es Instagram (4/4), Snapchat (3/4), Facebook (2/4) y Twitter (1/4). Por otro parte, en cuanto a la cantidad de plataformas virtuales por usuario, 1 de los 4 participantes es usuario de las 4 plataformas virtuales especificadas, y los restantes de dos.

Tabla $N^{\circ} 6$

Participantes y porcentajes uso de Plataformas virtuales utilizadas por los alters en la red personal.

\begin{tabular}{ccccc}
\hline Participantes & Facebook & Twitter & Snapchat & Instagram \\
\hline 1 & $100 \%$ & $65 \%$ & $91 \%$ & $97 \%$ \\
2 & $62 \%$ & $0 \%$ & $0 \%$ & $68 \%$ \\
3 & $0 \%$ & $0 \%$ & $85 \%$ & $96 \%$ \\
4 & $0 \%$ & $0 \%$ & $68 \%$ & $94 \%$ \\
\hline
\end{tabular}

Figura 1

$R P$ de los participantes.
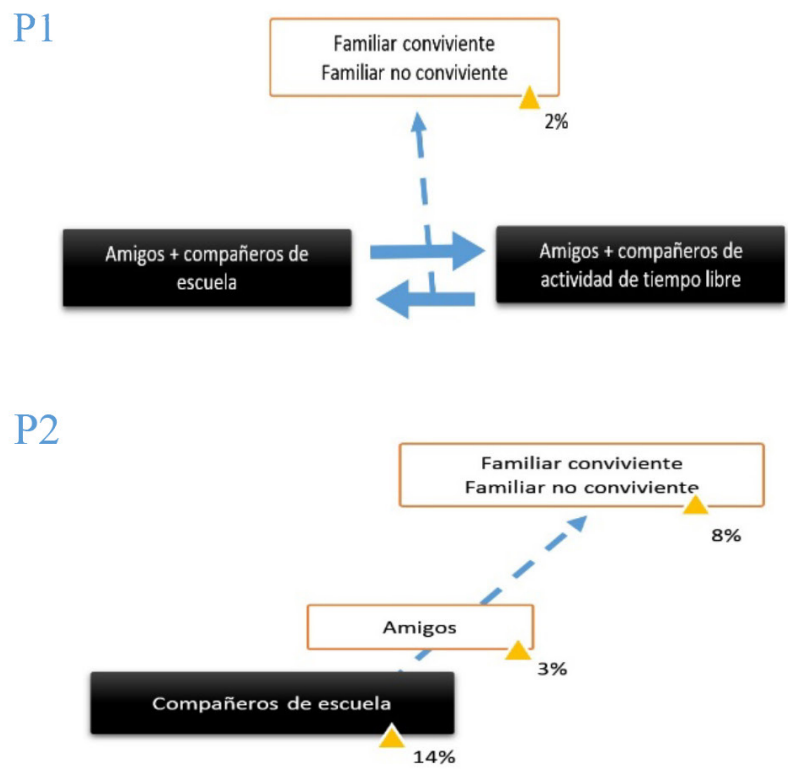

Los números presentados en la tabla precedente son el producto de los porcentajes extraídos del software Egonet a partir del cuestionario aplicado. Estos porcentajes pertenecen a los 35 alters nombrados por cada participante, señalados como usuarios de alguna de las cuatro plataformas virtuales indagadas.

En primer lugar, la plataforma virtual Instagram presenta el mayor porcentaje de uso, a la cual siguen: Snapchat, Facebook y Twitter. En segundo lugar, en cuanto a puntuaciones extremas, una de las plataformas virtuales tiene el $100 \%$ de usuarios por parte de la red personal es Facebook en P1. Snapchat, Twitter y Facebook no tienen usuarios en algunas redes personales de participantes. En tercer lugar, en cuanto a plataformas virtuales, hay coincidencia entre los participantes y su red personal.

Características estructurales y composicionales de las RP A continuación, se presentan esquemas de redes personales que sintetizan el grafo resultante del análisis de Egonet. Cada esquema es caracterizado por: las densidades de subgrupo y de conexión, el rol predominante del subgrupo y el porcentaje de no usuarios de Instagram. La densidad de subgrupo sería la parte que presenta mayor conexión en el componente, y el nivel de interconexión entre esa parte. La densidad de conexión es el nivel de vínculo entre los subgrupos previamente identificados. El rol predominante del subgrupo es el tipo de relación que tienen ese grupo con el entrevistado/a. El porcentaje de no usuarios de Instagram: se toma como referencia esta plataforma virtual ya que es la más usada por los/as entrevistados/as y se identifica este subgrupo con el porcentaje de no usuarios que existe en ella.

La RP del participante1, tiene dos subgrupos, liderados por compañeros/as de actividad en tiempo libre y compañeros/ as de escuela. En cada subgrupo, el participante estableció niveles de relación donde el mayor nivel es de amigos/as y menor conexión con los familiares.

Los no-usuarios de Instagram le pertenecen a los familiares (2\%).

La RP del participante2 tiene la menor densidad en relación a las demás RP. El subgrupo que tiene mayor densidad es compañeros de escuela y le sigue amigos y familiares. Establecen una conexión lábil entre todo el componente donde los amigos son el nexo entre familiares y compañeros de escuela. Tiene la mayor cantidad de no-usuarios de Instagram en relación a los demás participantes. 

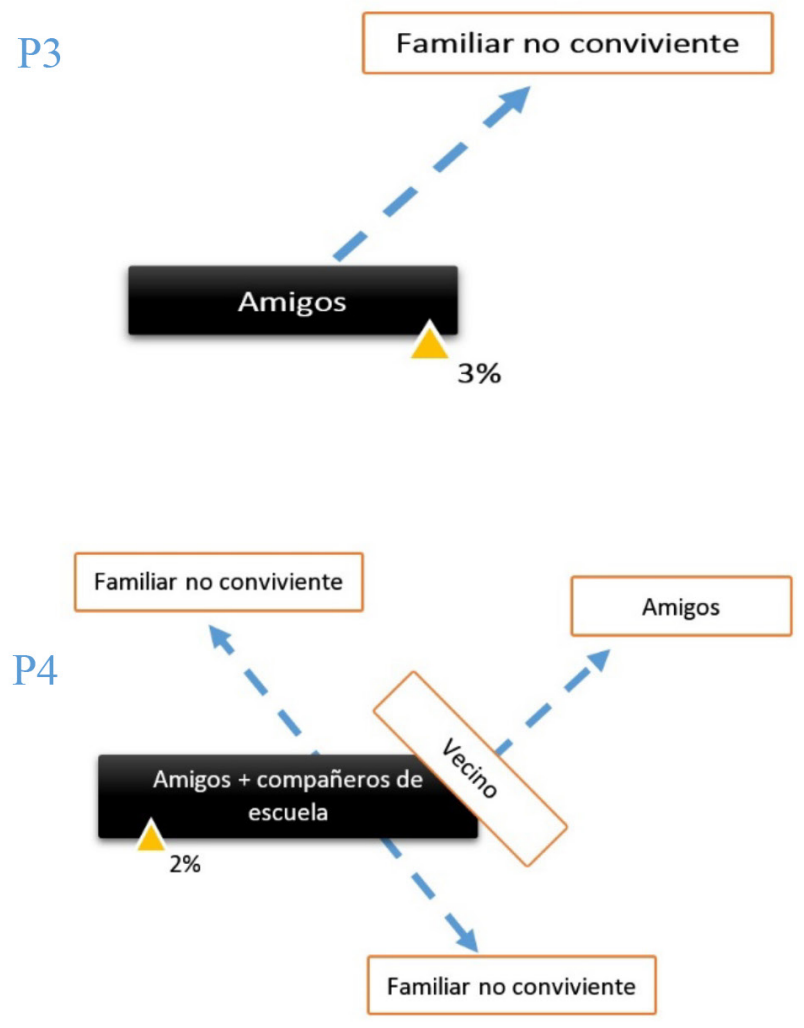

La RP del participante3 posee solo 1 familiar y lábilmente conectado con subgrupo amigo. Los amigos están fuertemente conectados, poseen mayor densidad y se encuentran los no-usuarios de Instagram.se encuentran los no-usuarios de Instagram.

La RP del participante4 tiene diversidad de relaciones a diferencia de los anteriores. Los subgrupos se encuentran lábilmente conectados.

El subgrupo con mayor densidad es amigos+compañeros de la escuela. vecinos intermedian entre dos subgrupos. Los familiares están conectados a distintas partes del subgrupo más denso.

Los no-usuarios de Instagram se encuentran en el de mayor densidad.

Las RP de los/as cuatro entrevistados/as están internamente conectadas con diversidad de densidades, pero en un solo componente . Instagram es identificada por los adolescentes como la más usada, por lo que podemos afirmar que existe un gran porcentaje de miembros de la RP de los entrevistados con Instagram (ver tabla No 6). Las RP en su mayoría tienen: menos del $19 \%$ de familiares, son redes jóvenes (según la edad promedio) y con predominancia de relaciones de pares.

\section{Referencias}

Densidad del subgrupo

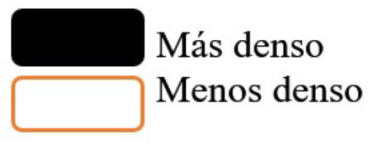

Densidad de conexión

$\longrightarrow$ más conectado
$\longrightarrow$ menos conectado
No usuarios de Instagram

porcentaje de no-usuarios de instagram

Tabla $\mathrm{N}^{\circ} 7$

Edad, género y rol de los alters.

\begin{tabular}{|c|c|c|c|c|c|c|c|c|}
\hline \multirow{3}{*}{ Participantes } & \multirow{3}{*}{$\begin{array}{c}\text { Edad } \\
\text { promedio } \\
\text { de la red } \\
\text { personal }\end{array}$} & \multirow{3}{*}{ Género } & \multicolumn{6}{|c|}{ Rol del alter en relación al participante } \\
\hline & & & \multirow{2}{*}{$\begin{array}{l}\text { Compañero/a } \\
\text { de escuela }\end{array}$} & \multicolumn{2}{|c|}{ Familiar } & \multirow[b]{2}{*}{ Vecino } & \multirow[b]{2}{*}{ Amigo } & \multirow{2}{*}{$\begin{array}{l}\text { Actividad de } \\
\text { tiempo libre }\end{array}$} \\
\hline & & & & $\begin{array}{c}\text { No } \\
\text { conviviente }\end{array}$ & Conviviente & & & \\
\hline $\mathrm{P} 1$ & 18 años & Fem $74 \%$ & $20 \%$ & $3 \%$ & $10 \%$ & $0 \%$ & $45 \%$ & $22 \%$ \\
\hline P2 & 23 años & Masc $71 \%$ & $57 \%$ & $2 \%$ & $11 \%$ & $0 \%$ & $25 \%$ & $5 \%$ \\
\hline P3 & 16 años & Fem $51 \%$ & $0 \%$ & $3 \%$ & $0 \%$ & $0 \%$ & $97 \%$ & $0 \%$ \\
\hline P4 & 19 años & Fem 57\% & $22 \%$ & $11 \%$ & $8 \%$ & $5 \%$ & $54 \%$ & $0 \%$ \\
\hline
\end{tabular}

La tabla $\mathrm{N}^{\circ} 8$ presenta los datos referidos a la edad, género y rol de los alters de la red personal de cada participante. En relación con edad, se trata de un promedio realizado a partir de los 35 alters nombrados por cada participante. Por otro lado, el rol es la función social que el participante asigna al alter que nombró en el cuestionario del software Egonet.

La edad de la RP de los participantes está contenida en el intervalo de 16 a 23 años de edad. Esto demuestra que existe una gran cantidad de adolescentes, advirtiendo la importan- cia del grupo de pares en la etapa en que nos encontramos indagando.

El género femenino está mayormente representado en las $\mathrm{RP}$ liderando en 3 de 4 participantes. Sin embargo, en las RP de los P3 y P4 la diferencia está dada por poco porcentaje.

En cuanto al rol, la mayoría de los participantes puntúa más contactos en tres roles precisos: amigos/as, compañeros/as de escuela y actividad de tiempo libre. En cuanto el rol amigo, 
la excepción está dada en P2, en el que los/as compañeros de escuela tiene el mayor puntaje. El rol familia el mayor puntaje está dado en un $11 \%$ en P4.

Podemos decir que coinciden en los participantes la edad promedio de la RP (16 a 23 años), los roles que mayormente incluidos (amigos/as, compañeros/as de escuela y actividad de tiempo libre) y en las plataformas virtuales mayormente utilizadas (Instagram, Snapchat y Facebook). Se puede agregar, que las plataformas virtuales Instagram y Snapchat, tienen mayor éxito entre los adolescentes básicamente por la instantaneidad y el menor control parental. Básicamente, porque estas plataformas son menos utilizadas por los adultos. Esta afirmación se evidencia con mayor claridad a partir de los cambios devenidos ("las historias") a partir del surgimiento de Snapchat, lo que impactó en las demás redes como Facebook e Instagram.

\section{Las redes virtuales: lecturas intra-red.}

Las RV se construyeron a partir de la observación de las plataformas virtuales más utilizadas por cada participante. A continuación, se presentarán los resultados teniendo presente la descripción de la red virtual del participante y en segundo lugar, se expondrán las características de los nodos centrales o usuarios que tienen mayor importancia en la red.

Figura 2

Gráficos de la red virtual (RV) de cada participante.

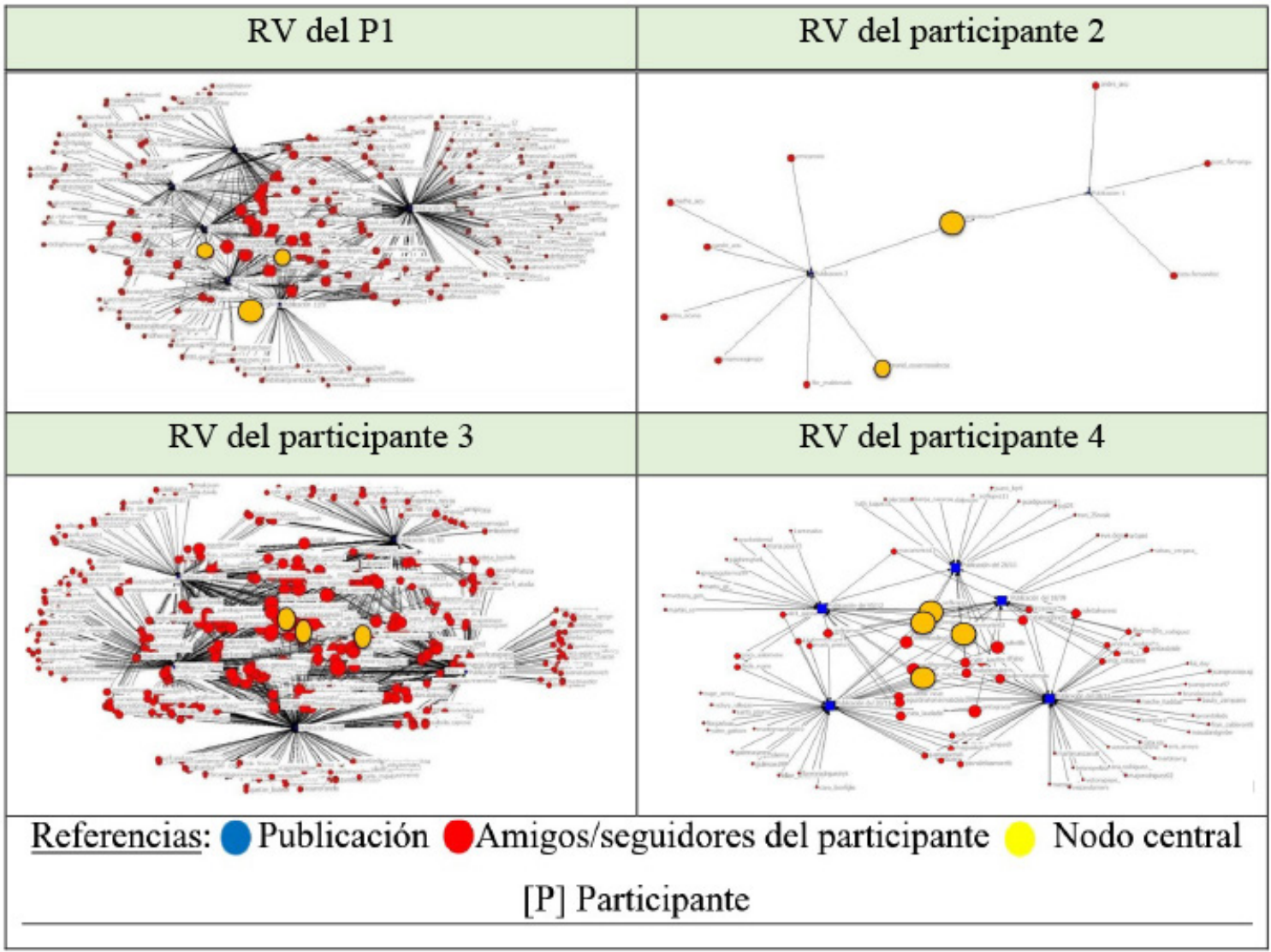

Las formaciones de las redes o reticulares demuestran diferentes dinámicas de acuerdo a la cantidad de publicaciones, MG-comentarios y seguidores recabados durante la observación. Las RV pertenecen a la interacción en la plataforma virtual Instagram.

El P1 es usuario de las cuatro plataformas virtuales. En cuanto a la estructura de la RV, las personas con mayor intensidad de interacción han puesto me gusta y han comentado en varias de las publicaciones del sujeto, sosteniendo así un mayor protagonismo entre las publicaciones. Elcentro del grafo existe alta densidad, lo cual indicaría que son varias las personas que repiten sus interacciones en diferentes publicaciones. En el polo contrario, las personas que interactúan con poca intensidad quedarían en la periferia de la RV y el número de estas personas también es alto.
La RV con menor densidad es la del P2, el cual en el término de 7 meses solo tiene 2 publicaciones. Indagamos a través de la entrevista semiestructurada y el sujeto refirió usar la plataforma virtual "para hablar con mis amigos, conectarme con mis compañeros y enterarme de algunas cosas" en lugar de realizar publicaciones. Además del bajo número de publicaciones, se notó un bajo número de interacción con estas publicaciones.

Por el contrario, la RV del P3 tiene la mayor densidad y se observa un núcleo fuertemente condensado, indicando que varias personas interactúan con más de una publicación en cinco puntos de fuga. Los seguidores de la RV que solo han participado una vez en las publicaciones están en la periferia de la RV. Adicional a la información precedente y desde un punto de vista metodológico, podemos agregar que el sujeto 
refirió usar Snapchat e Instagram con igual frecuencia, pero se decidió observar Instagram, ya que Snapchat tiene menos puntos de comparación con el resto de las redes sociales virtuales utilizadas por los participantes. Por ejemplo, no existe ningún equivalente del me gusta, no se pueden realizar comentarios públicos de las publicaciones, aunque sí se puede interactuar a través de conversaciones privadas que hubiesen sido difíciles de observar por la investigadora.

En la RV del P4, se observa en la parte central, a las personas que interactuaron con más de una publicación y en la periferia, a las personas que interactuaron solo con una publicación. En centro de la red no tiene alta densidad como la de los participantes 1 y 4, aunque se aprecia que tiene una distribución parecida de nodos.

\section{Las centralidades en la red personal (RP) y la red virtual (RV)}

La centralidad en una red o nodo central se define como aquel nodo que tiene mayor cantidad de conexiones en la red. A continuación, se presentarán los nodos centrales de las redes personales a través del rol y edad que proporcionó el participante. Luego, los nodos centrales de las RV situados en el gráfico de la Tabla $N^{\circ}$ 9. Por último, se exhibe la identificación del nodo central de la RP, con el objetivo de evaluar protagonismo en ambas redes.

Tabla $\mathrm{N}^{\circ} 8$

Nodo con mayor centralidad en la Red Personal (RP)

\begin{tabular}{ccc}
\hline Participante & Edad & Rol \\
\hline P1 & 16 & Amiga \\
P2 & 15 & Compañero de escuela \\
P3 & 16 & Amigo \\
P4 & 43 & Familiar Conviviente \\
\hline
\end{tabular}

En la tabla $\mathrm{N}^{\circ} 10$ se puede detallar que la mayoría de las centralidades es amigos de entre 15-16 años, a excepción de la P4, en donde el nodo central es ocupado por un adulto (familiar conviviente).

A continuación, se presentan datos sobre nodos centrales, surgidos a partir de la entrevista semidirigida a los participantes:

P1 al indagar acerca de este alter, la entrevistada nos explica que tiene un vínculo de amistad bastante cercano con ella, refiriéndolo como: "la mejor mejor amiga de todas".

P2 el entrevistado menciona que es un compañero de escuela y los miembros con mayor centralidad son de género masculino y de edades similares a la del sujeto.

P3 El participante afirma que esta persona es compañero del colegio desde los tres años de edad, del grupo de "los más amigos", y que, por lo tanto, "se conocen de toda la vida".

P4 La familiar conviviente es su madre y tiene "mucha relación y conoce a todos" sus contactos.

En la tabla $\mathrm{N}^{\circ} 11$ se presentan el rol de los nodos centrales que aparecen como puntos amarillos en la Tabla $N^{\circ}$ 9. Se consultó, a través de la entrevista semidirigida, sobre el rol y la identificación del nodo de la RV en la RP.
Tabla $N^{\circ} 9$

Rol del nodo central en la Red Virtual (RV) e identificación del participante en la Red Personal (RP)

\begin{tabular}{clc}
\hline Participante & \multicolumn{1}{c}{$\begin{array}{c}\text { Rol del nodo central } \\
\text { de la RV }\end{array}$} & $\begin{array}{c}\text { Identificación } \\
\text { en RP }\end{array}$ \\
\hline 1 & $\begin{array}{l}\text { 1. Compañero/a de escuela } \\
\text { 2. Compañero/a de escuela } \\
\text { 3. Compañero del club }\end{array}$ & Identificados \\
\hline 2 & $\begin{array}{l}\text { 1. Hermana } \\
\text { 2. Tía }\end{array}$ & $\begin{array}{c}\text { Identificado } \\
\text { No identificado }\end{array}$ \\
\hline 3 & $\begin{array}{l}\text { 1. Amigo de la infancia } \\
\text { 2. Amiga }\end{array}$ & No identificado \\
& 3. Amiga & Identificado \\
& 1. Hermana de un amigo & No identificado \\
& 2. Amiga & Identificadas \\
& 3. Amiga & \\
\hline
\end{tabular}

En primer lugar, los roles que aparecen con mayor asiduidad son: amigo/a, compañero de escuela y conocidas de la edad de los entrevistados/as. El rol familiar aparece como nodo central en tres de los participantes como: tía, hermana y prima. En segundo lugar, los nodos centrales en la RV no siempre están presentes en RP. Asimismo, muchos de los identificados no ocupan un lugar central en sus redes personales.

\section{Discusión}

El presente trabajo buscó puntos en común entre dos tipos de formaciones reticulares surgidas a partir de las relaciones cotidianas cara a cara y en las plataformas virtuales de las cuales los participantes del estudio son usuarios. Estos puntos de convergencia se analizaron desde la conceptualización de un contexto cibercultural en la etapa del desarrollo en la que se encuentran los participantes, la adolescencia. Se pretendió una aproximación al propósito de estudio a través de una metodología mixta, donde el tipo de estudio es de caso, y la representatividad es sustantiva, al leerse desde las categorías ofrecidas por los participantes.

En un primer nivel de discusión, algunas de las valoraciones que esgrimen los participantes sobre el uso de las plataformas virtuales se concentran en los beneficios del "estar conectados", la "rapidez y sencillez" en el uso y "la variedad de información transmitida". Estas valoraciones representan para los adolescentes un incentivo para la comunicación (Courtois, 2012; Vidales-Bolaños y Sádaba- Velazquer, 2017), los intercambios de diferentes tipos de contenido (Paredes et al., 2013) y un modulador del tipo de apoyo social (Linne, 2014).

En segundo lugar, podemos afirmar que las plataformas virtuales no tienen el mismo nivel de popularidad. Los resultados muestran que la plataforma virtual más utilizada por los adolescentes es Instagram y luego Facebook y Snapchat, y en último lugar Twitter. Dichos hallazgos, no están en concordancia con los estudios de Ak et al. (2012) y Paredes et al. (2013) en las que Facebook era la red social mayormente utilizada para conectarse entre adolescentes. En línea con lo anterior, Pérez (2018) plantea que hay creciente uso de plataformas vir- 
tuales (Instagram/Snapchat) con mayor exposición social.

La cibercultura adolescente puede indagarse operativamente a través de la actividad del adolescente en el uso de la plataforma virtual (Peter, 2005) y la influencia del contexto social en el sujeto (Linne, 2014), tanto para una interacción intensa como para interacción laxa moldeados por las relaciones próximas. El caso del P2 (15 años) en donde participación virtual fue de 2 publicaciones en 7 meses, las cuales obtuvieron 15 likes y quienes más "linkearon" fueron dos familiares. A su vez, al indagar los miembros de su RP, se demuestra que también poseen un nivel bajo de uso de las plataformas virtuales en las que se encuentran como usuario. Es decir, que es una $\mathrm{RP}$ coherente con el participante y con las actividades del mismo en plataformas virtuales. Sin embargo, no se priva a si mismo del uso sino que existe una mínima interacción debido a que las utiliza como manera de socializar y para consumo de información. En síntesis, hay un modo de uso y consumo de lo virtual, es parte de la interacción social del participante y es un patrón devenido de sus relaciones próximas.

Para describir la digitalización/virtualidad de las vinculaciones como modos de relacionarse y comunicarse (Paredes et al., 2013; Linne, 2014; Vidales-Bolaños y Sádaba- Velazquer, 2017), y de pertenecer en el contexto cibercultural del adolescente, podemos considerar a P1. Dicha participante (16 años) tiene la mayor cantidad de publicaciones en el menor tiempo (2 meses), siendo usuaria de todas las plataformas virtuales mencionadas y concordando dicha pertenencia con los miembros de su red personal. Asimismo, esta última red está diversificada en vínculos de pares de distintos ámbitos (club/ escuela) en donde la edad promedio de la RP es 18 años, es decir, mayoría de edad en Argentina. Hay conciencia de la centralidades de las redes (virtual-personal) en cuanto a su identificación y correspondencia red virtual y de la red personal. Por lo tanto, hay un alto nivel de apoyo social que se esgrime en las características composicionales y estructurales de su RP. Este engarce expresado entre RP y RV, plantea lo paradójico de dicotomizar lo offline y online en las relaciones como afirma Courtois et al. (2012), profundizar esta paradojo contribuiría a invisibilizar o no comprender las prácticas contemporáneas de vincularse.

La emergencia de nuevas plataformas virtuales brinda refugios para la intimidad adolescente junto a su grupo de pares (Giró, 2011; Margulis, 2004; Ak et al., 2012). Los familiares de los participantes no aparecen como centrales ni en la RP, ni en la RV y no superan el $17 \%$ de la red personal de los participantes. Esto se puede observar en el participante 3 de 16 años de edad, donde la red personal está compuesta por un $97 \%$ de amigos sin distinción de otro rol más que un familiar no conviviente. Esa ilusión de vivencia entre pares se afirma en la edad promedio de los integrantes de su red de 16 años y en la centralidad en su RP misma edad y género. En cuanto a la RV existe gran cantidad de intercambios a través de sus publicaciones (1055 entre comentarios y likes); los tres centrales de su RV son adolescentes de la misma edad.

Las centralidades en las redes están determinadas por amigos y conocidos en mayoría de las redes personales y en un solo caso, es familiar. En las redes virtuales no hay demasiadas diferencias, aunque hay mayor presencia de familiares no convivientes. No hay coincidencia al realizar el entrecruzamiento entre las centralidades de redes virtuales y redes per- sonales, y muchos de los centrales en las RV no se identifican en las RP. Podríamos decir que las centralidades responden a: i. los objetivos de cada una de las redes en cuestión, ii. a las comunidades en las que se circunscriben y iii. a la dinámica particular de cada una.

Reflexionando sobre posibles líneas de investigación, resulta necesario ahondar en las centralidades de las redes tanto virtual como personal, los niveles de intercambio generados en las plataformas virtuales y ahondando en las prácticas y tecnologías digitales y los modos en que estas son apropiadas/ incorporadas.

A modo de conclusión, nos preguntamos si existen en la actualidad, vínculos o intercambios relacionales en esta etapa del desarrollo que no estén mediados por plataformas virtuales. En general los participantes, a la hora de proveer los nombres para la red personal, observaron sus smartphones para identificar a quién nombrar como alter. La posibilidad de un afuera y un adentro, nos aproxima a las discusiones sobre natura-nurtura o individuo-sociedad. Sin embargo, ¿cuál sería el oxímoron de lo virtual? En la cibercultura adolescente las divisiones no tienen lugar y las prácticas se renuevan en cada avance tecnológico y cultural. ¿Qué existiría por fuera de lo virtual en la adolescencia actual?

\section{Referencias bibliográficas}

Ak, S., Koruklu, N., y Yılmaz, Y. (2012) A Study on Turkish Adolescent's Internet Use: Possible Predictors of Internet Addiction. Cyberpsychology, Behavior, and Social Networking. 16 (3). 1-5. DOI: 10.1089/cyber.2012.0255

Ávila Molero, J. (2015). Repensando la etnicidad y el transnacionalismo desde el análisis de redes personales. . Redes. Revista Hispana Para El Análisis De Redes Sociales, 26(2), 158-170. DOI: http://dx.doi. org $/ 10.5565 / \mathrm{rev} / \mathrm{redes} .585$

Borgatti, S.P., Everett, M.G. and Freeman, L.C. (2002). Ucinet for Windows: Software for Social Network Analysis. Harvard, MA: Analytic Technologies.nalysi

Casquero, O. (2013). Composición y estructura de redes personales en entornos de aprendizaje personales. En L. Castañeda y J. Adell (Eds.), Entornos Personales de Aprendizaje: claves para el ecosistema educativo en red. Alcoy: Marfil.

Castañeda, L. y Gutiérrez, I. (2010). Redes Sociales y otros tejidos online para conectar personas. En Castañeda, L. (Coord.): Aprendizaje con Redes Sociales. Tejidos educativos en los nuevos entornos. Sevilla: MAD Eduforma

Courtois, C., All, A. \& Vanwynsberghe, H. (2012) Social Network Profiles as Information Sources for Adolescents' Offline Relations. Cyberpsychology, Behavior, And Social Networking. 15 (6). 290-296. DOI: 10.1089/cyber.2011.0557

Evans, D. (2011). Internet de las cosas. Cómo la próxima evolución de Internet lo cambia todo. Cisco Internet Bussiness Solutions Group-IBSG, 11(1), 4-11.

Giró, J. (2011). Las amistades y el ocio de los adolescentes, hijos de la inmigración. Papers: revista de sociología, 96 (1), 77-95. Recuperado de: http://www.academia.edu/7987496/Las_amistades_y_el_ocio_de_ los_adolescentes_ 
Hanneman, R. A., \& Riddle, M. (2003). Introduction to social network methods. Riverside, CA: University of California, Riverside.

Hernández Sampieri, R., Fernández Collado, C., \& Baptista Lucio, P. (2014). Metodología de la investigación. Sexta Edición. Editorial Mc Graw Hill. México.

Instagram Inc. (2016). Instragram (Version 10.3). (Aplicación móvil). Descargado de https://itunes.apple.com/ ar/app/instagram/id389801252?mt $=8$

Jang, J. Y., Han, K., Shih, P. C., \& Lee, D. (2015). Generation like: Comparative characteristics in Instagram. Proceedings of the 33rd Annual ACM Conference on Human Factors in Computing Systems. 4039-4042. Recuperado de: http://pike.psu.edu/publications/ chi15.pdf

Lévy, P. (2007). Cibercultura. Informe al Consejo de Europa. México: Anthropos.

Linne, J. (2014). Usos comunes de Facebook en adolescentes de distintos sectores sociales en la Ciudad de Buenos Aires. Comunicar: Revista Científica de Comunicación y Educación, 22(43), 189-197.

Margulis, M. (2014). Adolescencia y Cultura en la Argentina. Perspectivas Metodológicas, 1(4): 1-7.

Martínez Carazo, P. (2006). El método de estudio de caso: estrategia metodológica de la investigación científica. Pensamiento \& Gestión, (20), 165-193.

McCarty, C. (2010). La estructura en las redes personales. Redes: revista hispana para el análisis de redes sociales, 19, 0243-271.

McCarty, C., 2011. EgoNet. Personal Network Software. Disponible en: http://sourceforge.net/projects/egonet/ Harvard, MA: Analytic Technologies.

Molina J. L., Lerner J., y Gómez Mestres, S. (2008). "Patrones de cambio de las redes personales de inmigrantes en Cataluña". Redes: revista hispana para el análisis de redes sociales, 15 (4):1-17.

Ortega Gutierrez, E. y Ricaurte Quijano, P. (2010). "Facebook o los nuevos rostros de la socialidad". Virtualis. 2. 72-85.

Paredes, A., Aguirre, J., Vitaliti, J. M., Strafile, S., Jara, C. y Sottile, C. (2015). "Redes Personales Digitales y Contexto Escolar. Estudio de un grupo de adolescentes de Mendoza (Argentina)". Estudios Sociales Contemporáneos. 12. 142 - 160.

Paredes, A., Vitaliti, J. M., Aguirre, J., Strafile, S. y Jara, C. (2013). "Tipos de apoyo y la digitalización de las redes personales. El uso de Facebook de adolescentes rur-urbanos de Mendoza (Argentina)". Redes, Revista Hispana Para El Análisis De Redes Sociales, 24(1), 97-123. DOl:10.5565/rev/redes.540

Pèrez, Vanessa (2018) Aproximación a la investigación psicológica en Internet y redes sociales. Summa Psicológica UST, 15(1), 98-105. DOI:10.18774/0719448x.2018.15.vp

Peter, J., Valkenburg, P. M., y Schouten, A. P. (2005). Developing a Model of Adolescent Friendship Formation on the Internet. Cyberpsychology, Behavior, And Social Networking. 8 (5), 423-431.

Rodríguez Treviño, J. (2013). Cómo utilizar el Análisis de Redes Sociales para temas de historia. Signos Históricos, (29), 102-141.

Snap Inc. (2016) Snapchat (Versión 10.34.1.1). (Aplicación móvil). Descargado de: https://itunes.apple.com/ar/ app/snapchat/id447188370? $\mathrm{mt}=8$
Twitter Inc. (2016). Twitter (Versión 6.69.1). (Aplicación móvil). Descargado de: https://itunes.apple.com/ar/ app/twitter/id333903271?mt=8

Urresti, M. (2008). Ciberculturas juveniles: los jóvenes, sus prácticas, y sus representaciones en la era del internet. Buenos Aires: La Crujia.

Vidales-Bolaños, M. J. y Sádaba-Velazquer C. (2017). "Adolescentes conectados: La medición del impacto del móvil en las relaciones sociales desde el capital social". Comunicar: Revista Científica de Comunicación y Educación, 53 (15), 19-28. 